\title{
Chapel of the Blessed
}

\author{
Capilla de los bienaventurados
}

Campos de refugiados; Plaza de San Pedro; etc.

\section{Proyecto destacado por el Comité Científico}

«Blessed are the poor in spirit, for theirs is the kingdom of heaven. Blessed are they who mourn, for they shall be comforted. Blessed are the meek, for they shall inherit the earth. Blessed are they who hunger and thirst for righteous-ness, for they shall be satisfied. Blessed are the merciful, for they shall obtain mercy. Blessed are the pure of heart, for they shall see God. Blessed are the peacemakers, for they shall be called children of God. Blessed are they who are persecuted for the sake of righteousness, for theirs is the kingdom of heaven» (Matthew 5:3-10).

A place for quiet and silence. Can an object question human indifference? The metaphor of the world's assembly? The primitive hut. Built with recycled leftover materials, enhancing primitive and industrial techniques. Two cylinders: a 4 meters high circular fence, made from a metal structure guides a weave of wooden brunches and makes room for an in-between space: the threshold of the 'inside'. The white room, a 6 meters cylinder built from recycled formwork panels, paved with earth, allows the gathering of love, suffering and hope. Around the Eucharistic supper and the 'table' of the Word, the assembly gives tangibility to the experience of Emaus.

https://doi.org/10.17979/aarc.2013.3.0.5112 

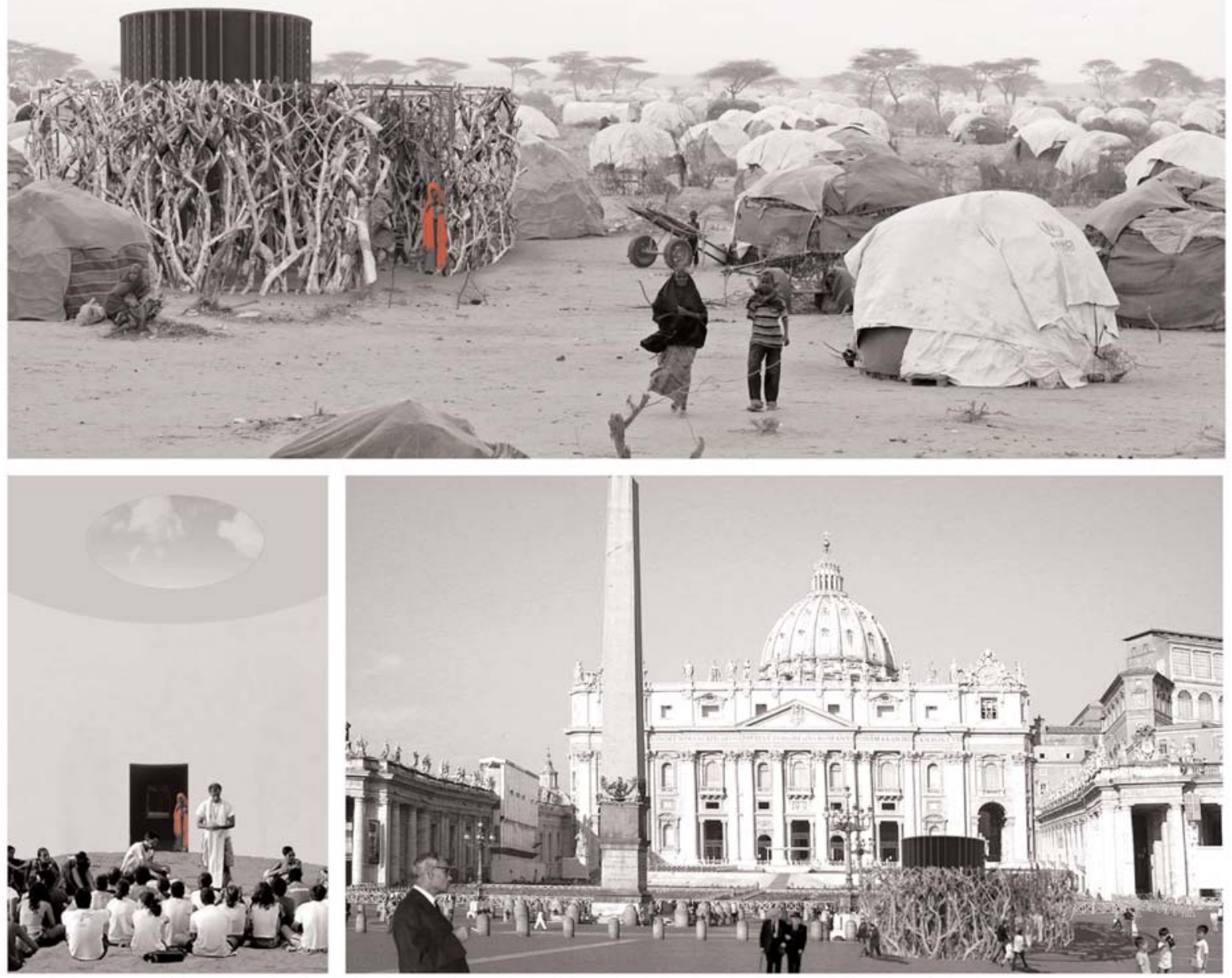

Blessed are the poor in spirit, for theirs is the kingdom of heaven. Blessed are they who mourn, for they shall be comforted. Blessed are the meek, for they shall inherit the earth. Blessed are they who hunger and thirst for righteous.

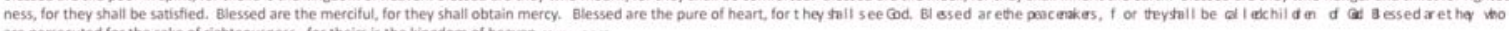
are persecuted for the sake of righteousness, for theirs is the kingdom of heaven. Mamms sas:

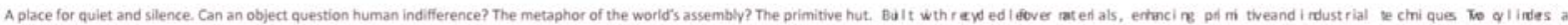

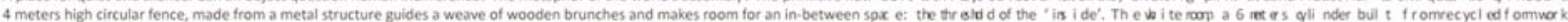
panels, paved with earth, allows the gathering of love, suffering and hope. Around the Eucharistic supper and the 'table' of the Vord the as $x$ tt y givest agi bili ty to the eperienced Eras.
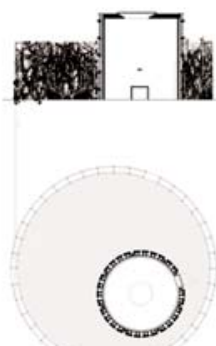

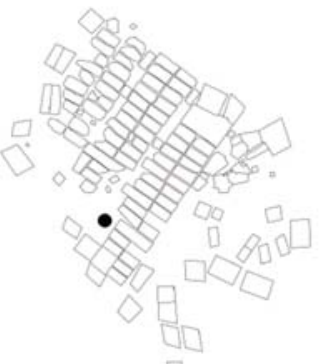

(7)

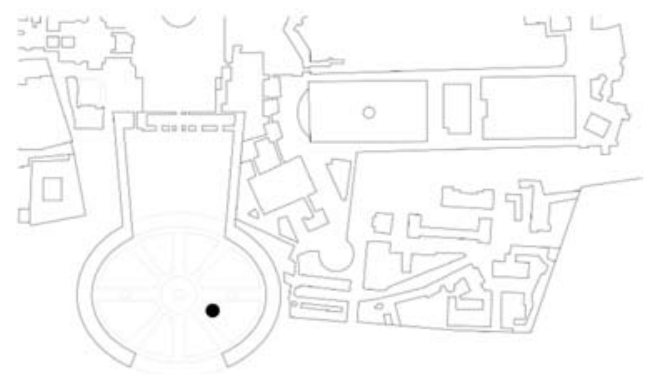




\title{
Re-ligados
}

\author{
Re-linked
}

T113 arquitectura - Eloi Arán Sala, Giuseppe Giacalone, Roger Panadès y María del Mar Viladot Múltiples ubicaciones

El término religión proviene del latín «re-ligare», que significa crear lazos de unión.

Partiendo del significado etimológico de la palabra y de la llamada de Jesús a sus primeros discípulos «Jesús les dijo: Seguidme, y yo os haré pescadores de hombres» (Mt 4,19), proponemos un espacio de oración y culto católicos formalmente reconocible con un gesto contundente, inspirado en el Ichtus.

Constructivamente se han pensado unos materiales para definir este espacio, inspirados en la imagen del pescador: una tarima de madera que hace de base - la barca - y una envolvente multiforme creada por dos capas curvas de red metálica - las redes - soportadas por postes.

El edificio, por su morfología flexible, permite dualidad en el uso: un espacio recogido en su interior, de tal manera que no sintamos envueltos por esa red en un espacio donde se puede estar, ver, oír, orar. En el exterior, en cambio, se genera un espacio que se abre y acoge para celebraciones de mayor aforo. El fácil acceso, con doble entrada, es una sutil transición del entorno urbano reforzado con la cruz.

El proyecto da respuesta a cualquier localización: tanto puede destacar en un centro urbano como se puede integrar en un parque. A su vez, si por exigencia de programa es necesario, se pueden crear diferentes formas de la envolvente usando el modulaje de la malla metálica de distinta forma.

Esta propuesta cumple así los requisitos de polivalencia, transportabilidad y capacidad icónica enunciados. 

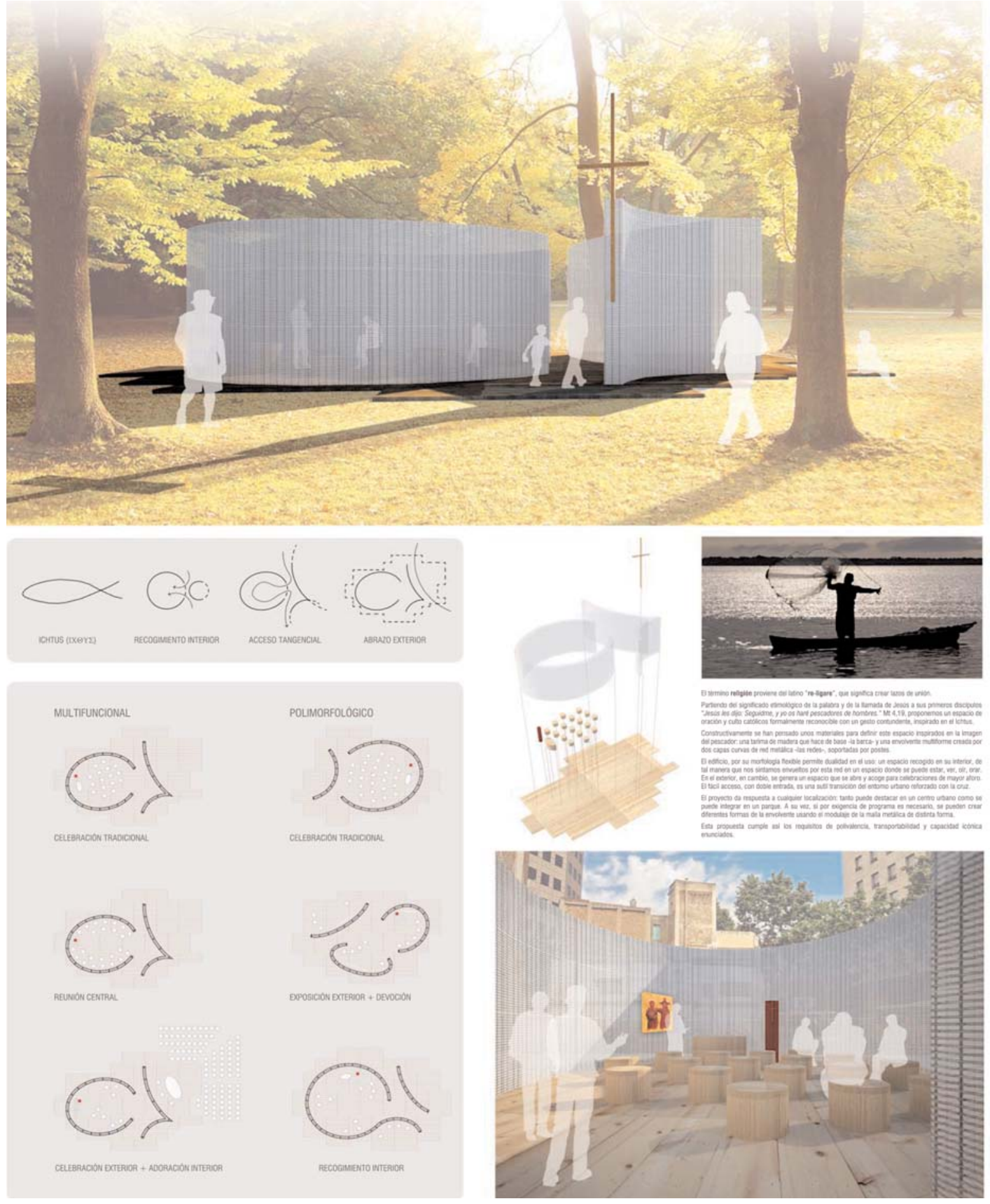


\title{
Capilla dolorosa para la Semana Santa
}

\author{
Dolorosa Chapel for Easter
}

TiA (Taller i Arte+Arquitectura) - José Luis Chacón Rodríguez + Nelson Gómez Callejas

Sevilla (España)

Siguiendo la convocatoria para diseñar un lugar de adoración al Santísimo, el TiA (Taller i Arte+Arquitectura) propone una instalación que integra arte y arquitectura como parte de las celebraciones de Semana Santa en Sevilla. Es una construcción en forma de capilla abierta o monumento, dedicada al dolor de —y en torno— a la Pasión de Jesucristo, a ser instalada y habitada en pleno durante el Jueves Santo.

Imaginando una nueva Confraternidad de Indias, dirigida y ejecutada por latinoamericanos, cuyos fines son agradecer la evangelización del continente americano y pedir por la unión y paz entre los pueblos iberoamericanos, la Capilla Dolorosa es el inicio y final de la $8^{a}$ procesión de Jueves Santo. Ubicada dentro de la fuente central de la Plaza América, de allí parte la procesión de costaleros y penitentes cargando los cuatro pasos del dolor (los palios de la Dolorosa y de los Ángeles Custodios, junto al ostensorio del Santísimo) en un circuito que los lleva hasta la carrera oficial y de vuelta. Estos pasos forman parte intrínseca de la capilla.

Construida en madera traída desde los trópicos americanos, la capilla se conforma de un único espacio interior, de planta en forma de rombo (antigua simbolización del Espíritu Santo), en cuyo centro se coloca la custodia (y donde se podría oficiar misa eventualmente, armando el altar y el ambón) y un puente conector (con la orilla de la fuente) para el acceso de los fieles. Dicho espacio puede albergar hasta doce personas de pie, aunque seis en actitud adorativa es el número ideal.

El cuerpo de la capilla lo componen: dos pares de cerramientos verticales ligeramente inclinados hacia afuera, hechos en bahareque (alma de caña brava, revestida de barro); y un entrepiso de madera, sobre el nivel de agua de la fuente. Estos elementos se apoyan sobre una estructura de cercos y rolas de madera. Los soportes más notorios son las seis rolas exteriores en posición de contrafuertes, que atraviesan los cerramientos verticales.

El puente conector, que cruza toda la construcción, está hecho de tablones separados entre sí (para dejar ver la superficie de agua), apoyados sobre un único cerco, que forma parte de la estructura global.

Lo plástico se une a lo tectónico en puntos focales clave del espacio sacro: el palio de la Dolorosa en la punta norte del rombo, como fondo visual de la liturgia; los bajorrelieves del Nazareno sobre la superficie interna del cerramiento vertical, acompañando a los fieles; los palios de los Ángeles Custodios en la punta de acceso, que resguardan el recinto; y el ostensorio dorado apoyado sobre la estructura umbilical del centro del espacio interior, al cual todas las miradas y deseos están dirigidos. 


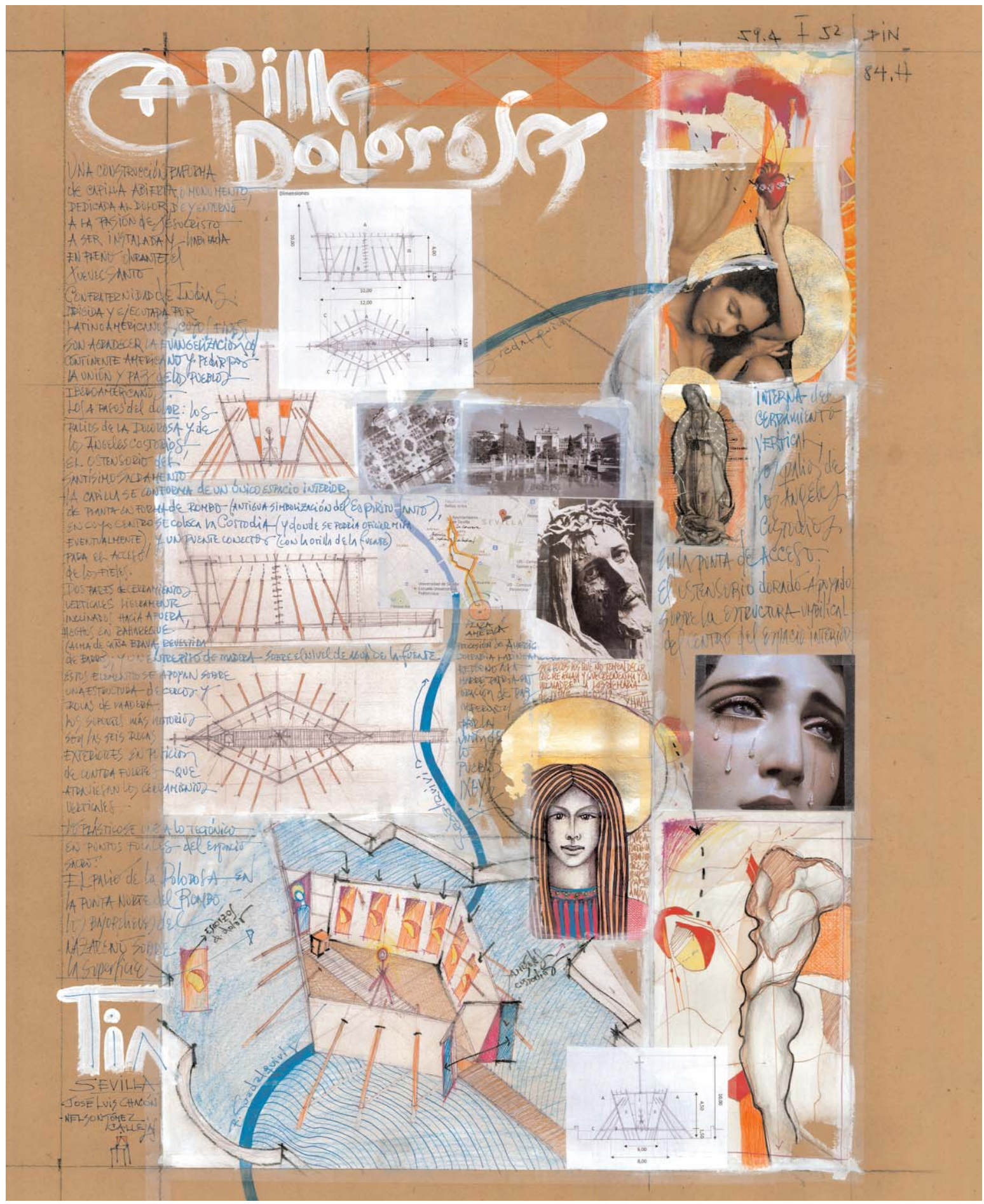




\title{
Un pequeño espacio de silencio y oración
}

\author{
A tiny space of silence and prayer
}

Tino Grisi + Angelo D'Apolito

Sevilla (España) y otras localizaciones

El proyecto se basa en la exposición del Santísimo Sacramento en la ciudad. Como sucede en la procesión del Corpus Christi, la custodia con la Forma consagrada se coloca bajo un baldaquino compuesto por cuatro columnas verticales libremente inclinadas y un plano dorado suspendido en el aire. El baldaquino funda el lugar de adoración, compuesto en torno al mismo como envoltorio que expresa el sentido de pasear, encontrarse y conversar allí donde todo lo que es profano encuentra significación religiosa, favoreciendo el encuentro viviente constituido por la presencia real de Jesucristo, su tienda en medio de nosotros.

El cándido refugio, compuesto por paneles de madera barnizados, atravesado por pequeños pilares metálicos, en el que paredes, suelo y cubierta se ligan sin solución de continuidad, está provisionalmente tamponado por superficies leves, [realizadas] en aluminio cepillado y pantallas de vidrio amarillo-bronce que dejan transparentar en lo alto el baldaquino, y frontalmente, la imagen del Sacramento hacia el exterior, mientras lo rodean de luz en el interior. Una ventanita cubierta se orienta hacia la Forma desde la parte contraria.

La disposición planimétrica se articula libremente en torno al altar sobre el que se encuentra la custodia, dando la posibilidad de dirigirse, sentarse y arrodillarse desde puntos y sectores diversos para una oración personal, o reunirse como pequeña asamblea para la celebración eucarística. Un espacio periférico de servicio se ha añadido para la necesidad de preparación de los celebrantes y manutención del ambiente. La superficie interna es de 38 m2; el área cubierta es de 57 m2. 


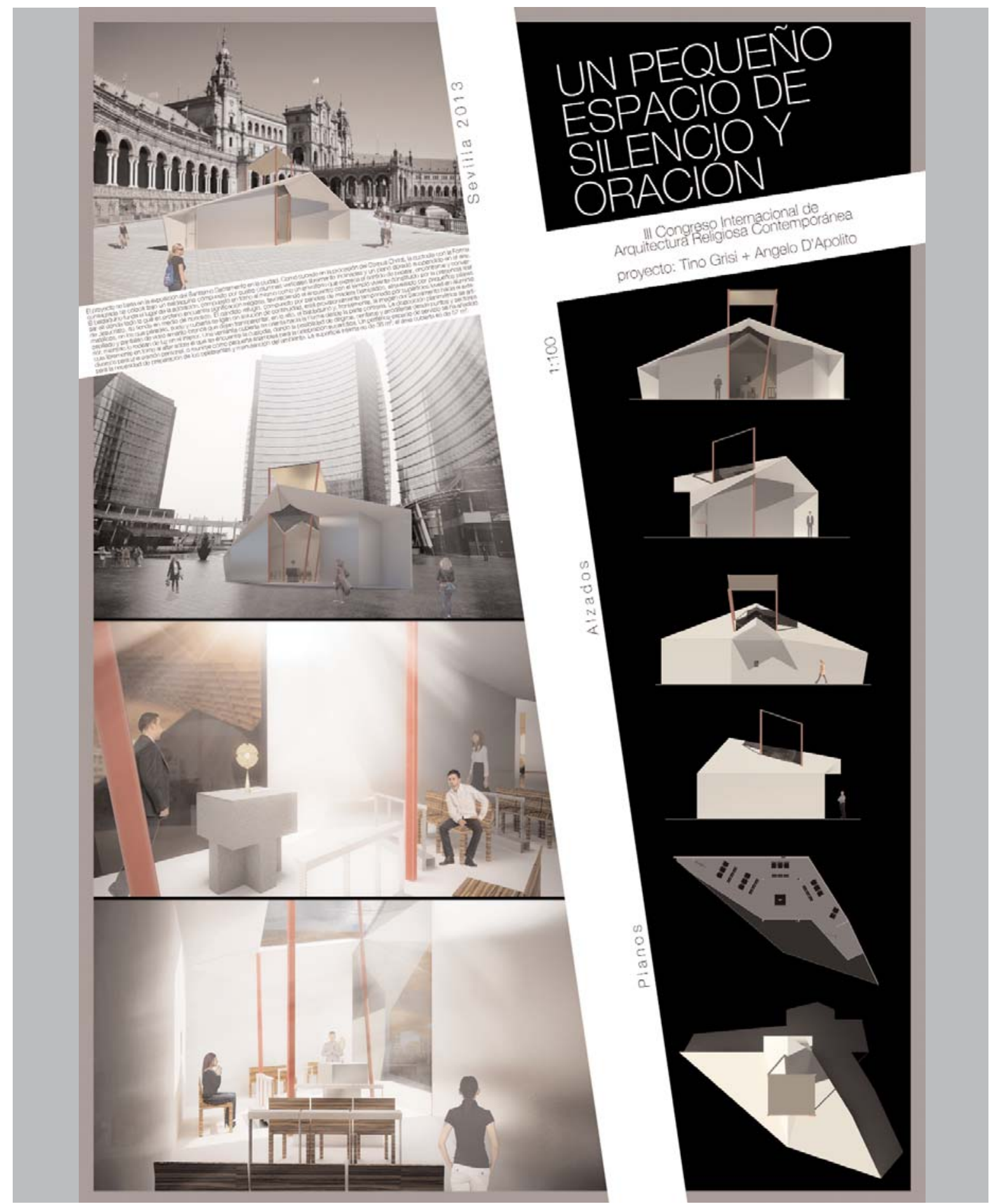




\section{Capilla del centro Vedruna. Hermanas Carmelitas de la Caridad (Vedruna)}

Vedruna Center Chapel. Carmelite Sisters of Charity (Vedruna)

Miguel Angel Santibáñez Llinás

Valladolid (España)

Se trata de un lugar de encuentro y práctica espiritual, en el Centro Vedruna, situado en el complejo denominado «Colegio Ave María» en Valladolid.

La capilla es un espacio sencillo y luminoso que se utiliza para celebraciones de pequeños grupos. Todos los elementos están pensados para generar un espacio que invite al recogimiento interior, la oración y la espiritualidad.

Una magnífica talla en madera de una Virgen orante del escultor Javier Martínez Pérez, protagoniza la capilla. La paz que transmite la figura de la Virgen impregna el espacio y le confiere su especial carácter. 


\section{CAPILLA DEL CENTRO VEDRUNA}

Arquitecto: Miguel Ángel Santibáñez Llinás

Se trota de un iugar de encuentro y próctica espittual, en at Centio vedruna, stuodo en el Complejo denominodo "Colegio Ave Maria" en Valodolid.

La caplla es un espacio sencilio y urninoso que se utiliza para celectrociones de pequenos grupos. lodos los etementios eston pensodos para generar un espocio que imbe al recogimiento intenot, la oración y la espit-

Una mognifica tolla en modera de una Vrgen cante del escullor Jovier Mortinez Pérez protogoniza la capila.

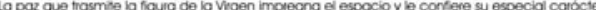

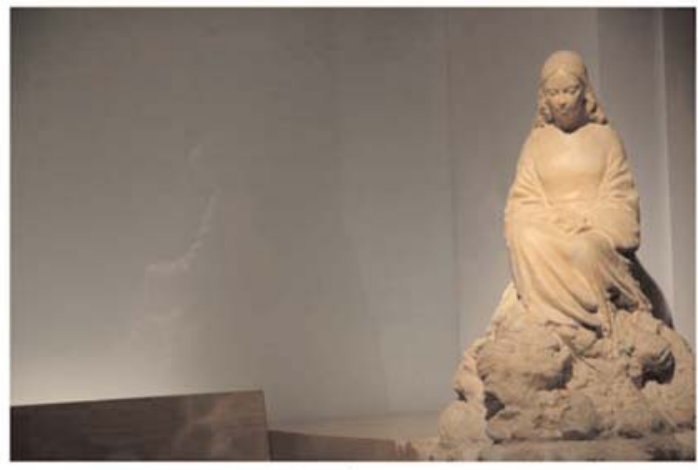

Virgen orante, Escultor: Javier Martínez Pérez
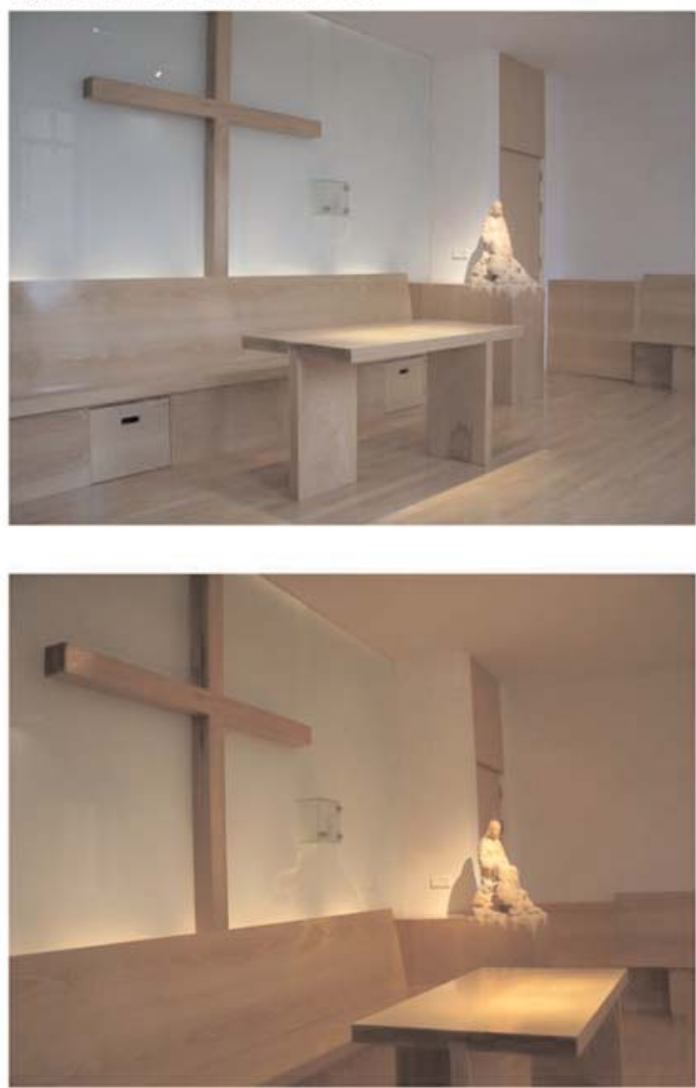
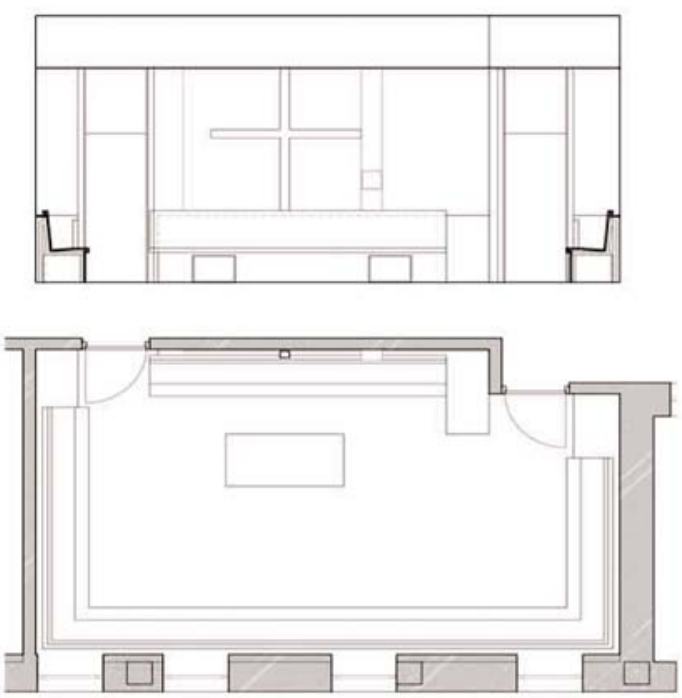

Sección y planta de la capilla del Centro Vedruna

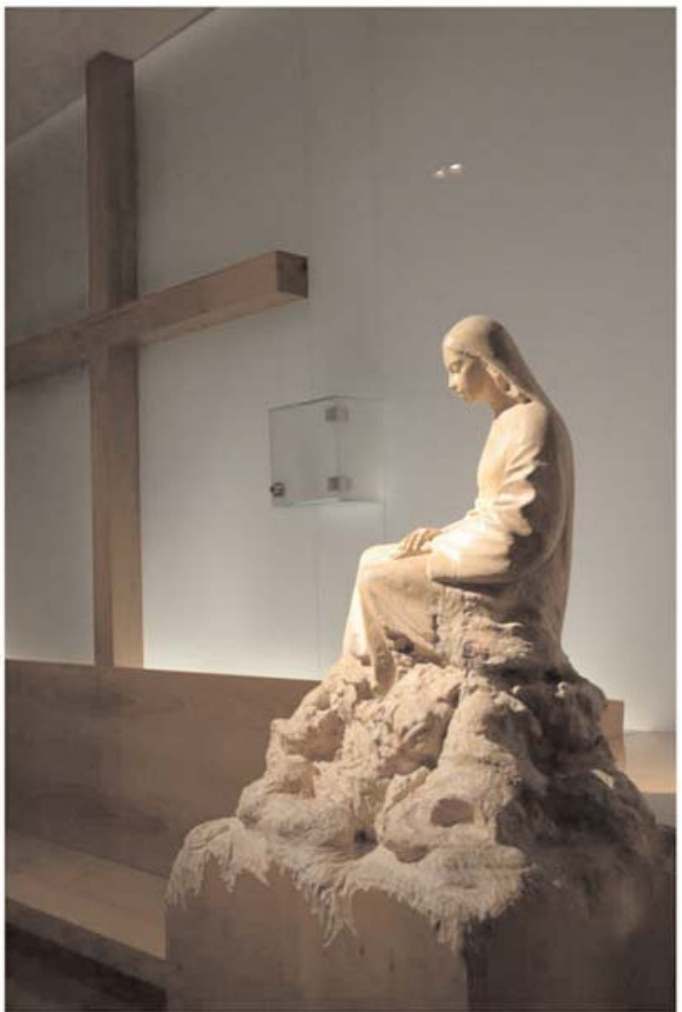




\title{
Pabellón del Silencio. Hermanas Carmelitas de la Caridad (Vedruna)
}

\author{
Pavilion of Silence. Carmelite Sisters of Charity (Vedruna)
}

Miguel Angel Santibáñez Llinás

Valladolid (España)

Se trata de una pequeña intervención de nueva planta en las inmediaciones del complejo «Colegio Ave María» e las HH Carmelitas de la Caridad de Vedruna, en Valladolid.

El Pabellón del Silencio se expresa con gran limpieza formal, muestra un aspecto cerrado y ensimismado que le confiere una imagen rotunda.

Su construcción en hormigón visto blanco, armoniza con el aplacado del edificio principal del complejo, no muy lejano, de piedra caliza de Campaspero.

El pabellón cuenta con un acceso principal en su fachada Este, formalizado por un gran voladizo/porche que comunica con el patio interior a través de otro porche que lo abraza en forma de L, en sus alzados Sur y Este. Este porche configura el segundo acceso al interior, desde el citado patio localizado en la fachada Norte.

El Pabellón del Silencio se caracteriza por un estudiado juego de volúmenes que confieren a los espacios interiores y exteriores una sencilla expresividad orientada a la meditación. 
PABELLON DEL SILENCIO

Arquitecto: Miguel Ángel Santibáñez Llinás
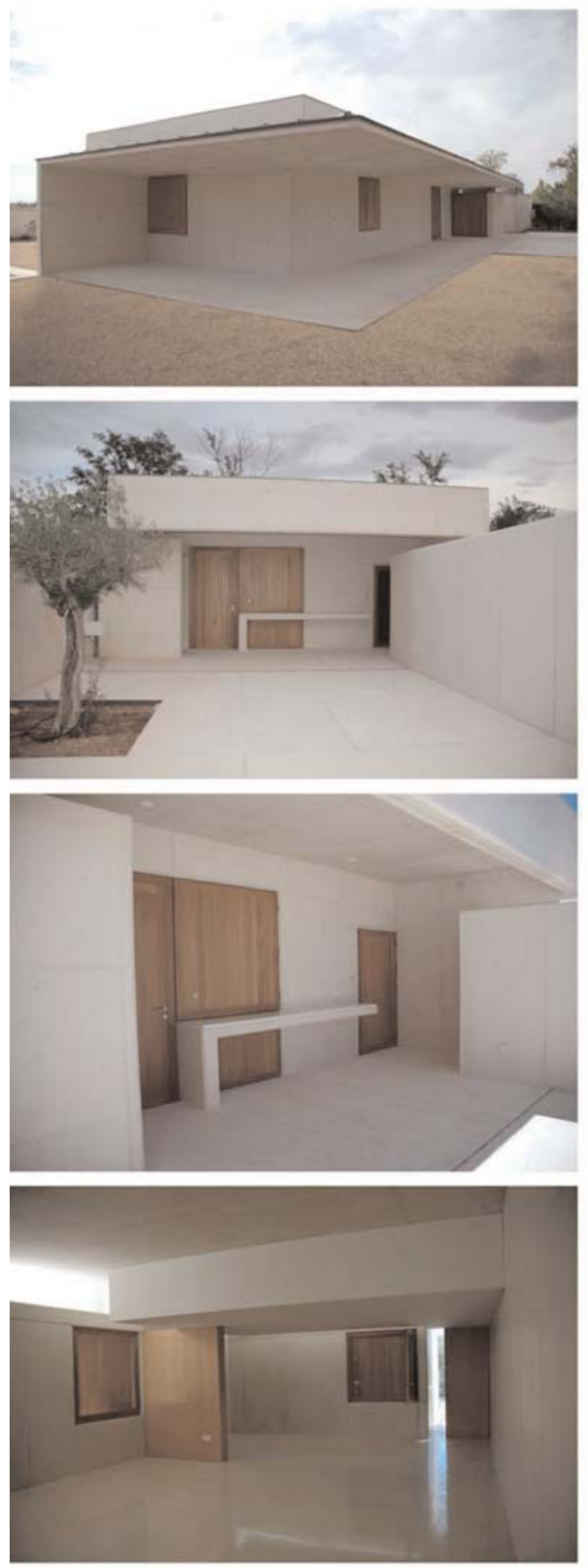
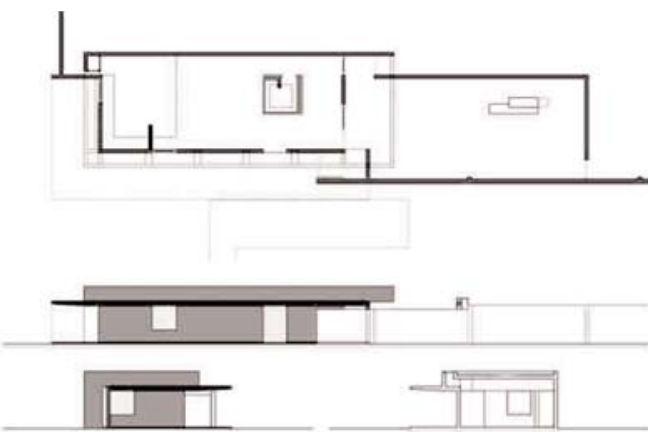

Planta, alzados y sección del Pabellón del Silencio

Se trota de una pequena intervención de nueva pianta en las inmediociones del complejo "Cologio

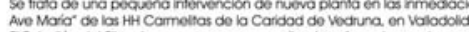

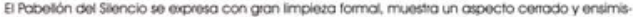
modo ave te contore una imogen totunda.

su constucción en hormigón visto bianca, armoniza con el aplocado dol odticio pencipal dol com.

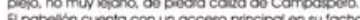

Este, formolizado por un gron

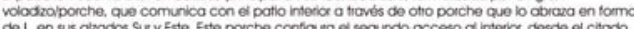
patio localzado en la fochoda Norte

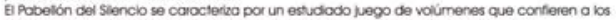

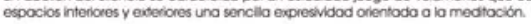
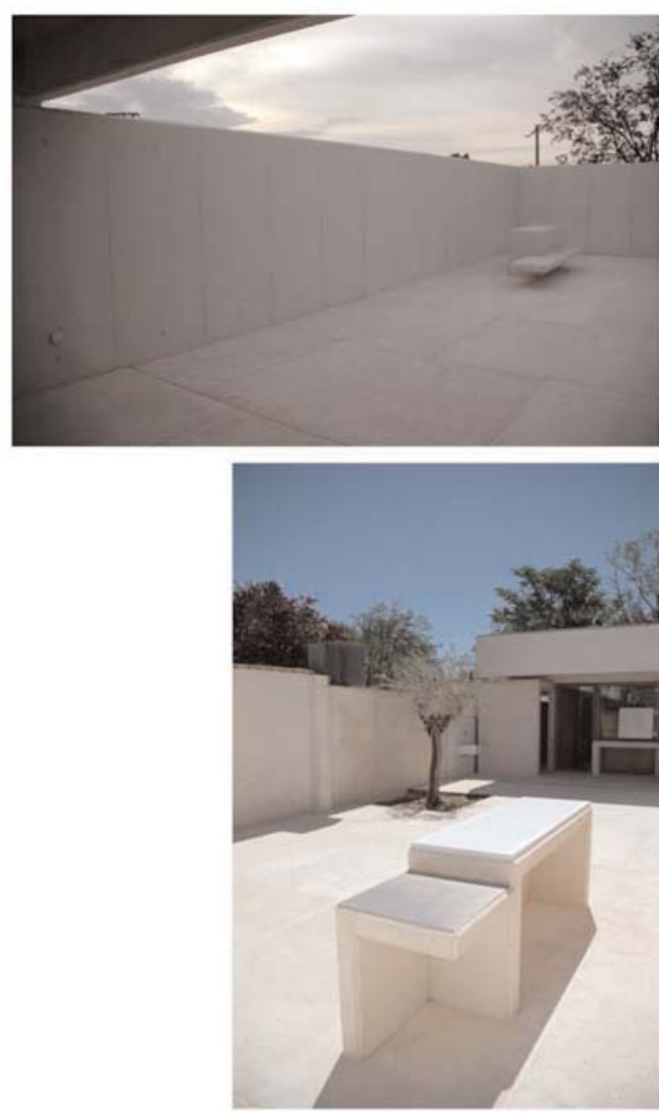


\title{
Saint John Baptist Chapel
}

\author{
Capilla de San Juan Bautista
}

Alejandro Beautell García

El Hierro, Canary Islands (Spain), 2013

The chapel is projected in a plan view as a single volume in a triangular shape, with an access from the opposite side of the acute angle of the triangle. This way, the space narrows in the plant and starts to increase in the height, as we approach the altar (from Latin, altare comes from altus «rise»), which constitutes the main element of the temple.

The life of Saint John Baptist, patron saint of the chapel, was an example of sobriety and austerity. John is portrayed as an ascetic figure, Jesus countered him with those who «are in royal palaces» and «wear fine clothes». The style of John the Baptist should urge all Christians to choose sobriety as a way of life. The same way as John defined himself as «the voice crying out in the desert», the chapel, that will bear his name, will also protest against the excesses of the past and will be the precursor of a new stream, testimony that a new religious art is possible.

Austerity was not just a moral conviction, it was a necessity. We use the materials we found in the island... we had no gold, but we had the light, we had no marble, but we worth concrete, there is not plaster filigrees, but plaster of tiroliano serves us, you will not find crystal chandeliers, but, when night falls, we will light up the bulbs likewise. 

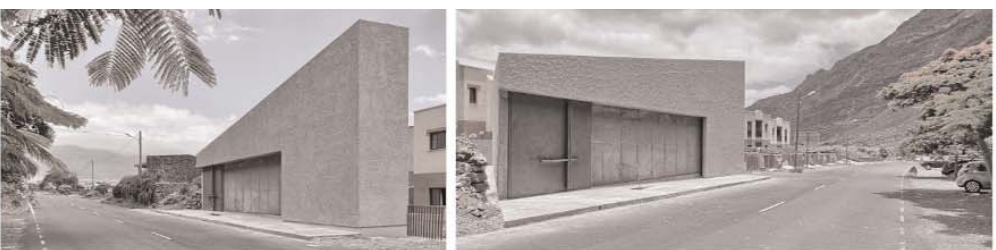

\section{Saint John Baptist Chapel}

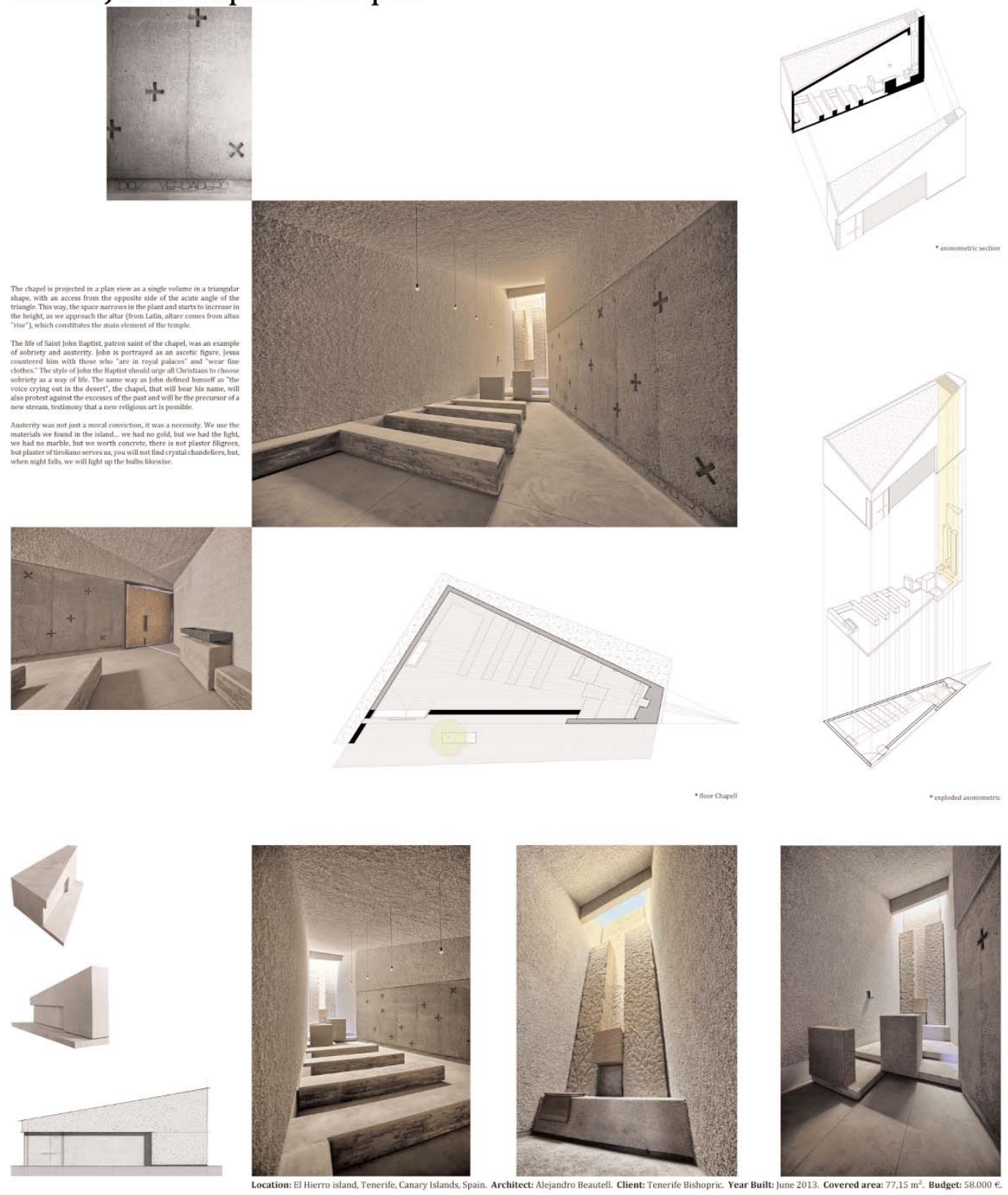

